\title{
Serial Transverse Enteroplasty (STEP): A Novel Bowel Lengthening Procedure
}

\author{
By Heung Bae Kim, Dario Fauza, Jennifer Garza, Jung-Tak Oh, Samuel Nurko, and Tom Jaksic \\ Boston, Massachusetts
}

\begin{abstract}
Background/Purpose: Bowel lengthening may be beneficial for children with short bowel syndrome. However, current techniques require at least one intestinal anastomosis and place the mesenteric blood supply at risk. This study seeks to establish the technical principles of a new, simple, and potentially safer bowel lengthening procedure.
\end{abstract}

Methods: Young pigs $(\mathrm{n}=6)$ underwent interposition of a reversed intestinal segment to produce proximal small bowel dilation. Five weeks later the reversed segment was resected. Lengthening of the dilated bowel then was performed by serial transverse applications of a GIA stapler, from opposite directions, to create a zig zag channel. A distal segment of equal length served as an in situ morphometric control. Contrast radiologic studies were performed 6 weeks later, and the animals were killed. Statistical comparisons were made by paired $t$ test with $P$ less than .05 considered significant.

Results: After bowel lengthening, all animals gained weight $(66.7 \pm 3.0$ [SD] $\mathrm{kg} v 42.5 \pm 3.5 \mathrm{~kg} ; P<.001)$ and showed no clinical or radiologic evidence of intestinal obstruction. Intraoperatively, immediately after serial transverse enteroplasty, the intestine was substantially elongated $(82.8 \pm 6.7 \mathrm{~cm} v$ $49.2 \pm 2 \mathrm{~cm} ; P<.01)$. Six weeks after surgery, the lengthened intestinal segment became practically straight and, compared with the in situ control, remained significantly longer $(80.7 \pm 13.1 \mathrm{~cm} v 57.2 \pm 10.4 \mathrm{~cm} ; P<.01)$. There was no difference in diameter between these segments $(4.3 \pm 0.7 \mathrm{~cm}$ $v 3.8 \pm 0.4 \mathrm{~cm} ; P$ value, not significant).

Conclusions: Serial transverse enteroplasty (STEP) significantly increases intestinal length without any evidence of obstruction. This procedure may be a safe and facile alternative for intestinal lengthening in children with short bowel syndrome.

J Pediatr Surg 38:425-429. Copyright 2003, Elsevier Science (USA). All rights reserved.

INDEX WORDS: Short bowel syndrome, bowel lengthening, serial transverse enteroplasty.
B OWEL LENGTHENING procedures may be beneficial for children who suffer from short bowel syndrome (SBS). A variety of surgical procedures have been used to treat SBS, ${ }^{1}$ including the combined tapering/lengthening procedures described by Bianchi $^{2}$ and Kimura and Soper. ${ }^{3}$ A prudent surgical approach along with careful medical management can result in freedom from parenteral nutrition, even in some of the most severely affected patients. In this report, we seek to establish the technical principles and safety of a novel bowel lengthening operation that we have termed the serial transverse enteroplasty (STEP) procedure. The STEP procedure is easy to perform, does not require any bowel anastomoses, can theoretically more than double the length of bowel, and can be performed either primarily or after a prior Bianchi procedure.

\section{MATERIALS AND METHODS}

The Harvard Medical School animal management program is sanctioned by the American Association for the accreditation of Laboratory Animal Care (AAALAC, file \#000009) and meets National Institutes of Health Standards as set forth in the Guide for the Care and Use of Laboratory Animals (National Research Council Publication, revised 1996).

\section{Surgical Manipulation}

All surgical procedures were performed under general inhalational anesthesia using $2 \%$ to $4 \%$ halothane (Halocarbon Laboratories, River
Edge, $\mathrm{NJ}$ ) and preoperative intravenous antibiotic coverage with $1 \mathrm{~g}$ of cefazolin (BMH Ltd, Philadelphia, PA). Six young domestic pigs underwent laparotomy and creation of a partial intestinal obstruction using a $55-\mathrm{cm}$ reversed jejunal segment. Both anastomoses were performed in one, full-thickness layer, with running 5-0 monofilament polypropylene (Surgipro, United States Surgical Corporation [USSC], Norwalk, CT). After recovery, all animals were fed ad libitum. Five weeks later, animals underwent a second operation in which the reversed jejunal segment was resected and intestinal continuity restored using a single anastomosis between the dilated proximal bowel and the normal distal bowel. Then equal length bowel segments proximal and distal to the anastomosis were marked using a 4-0 polypropylene suture. The STEP procedure then was performed within the marked, dilated bowel proximal to the anastomosis. A reusable 65-mm GIA stapler (USSC) was applied sequentially, from alternating and opposite directions, in transverse, partially overlapping fashion creating a zig

From the Departments of Surgery and Gastroenterology, Children's Hospital and Harvard Medical School, and Harvard Center for Minimally Invasive Surgery, Boston, MA.

Presented at the 33rd Annual Meeting of the American Pediatric Surgical Association, Phoenix, Arizona, May 19-23, 2002.

This research was supported by grants from the United States Surgical Corporation and The Children's Hospital Surgical Foundation.

Address reprint requests to Tom Jaksic, MD, PhD, Children's Hospital, 300 Longwood Ave, Fegan 3, Boston, MA 02115. Copyright 2003, Elsevier Science (USA). All rights reserved. 0022-3468/03/3803-0028\$35.00/0

doi:10.1053/jpsu.2003.50073 


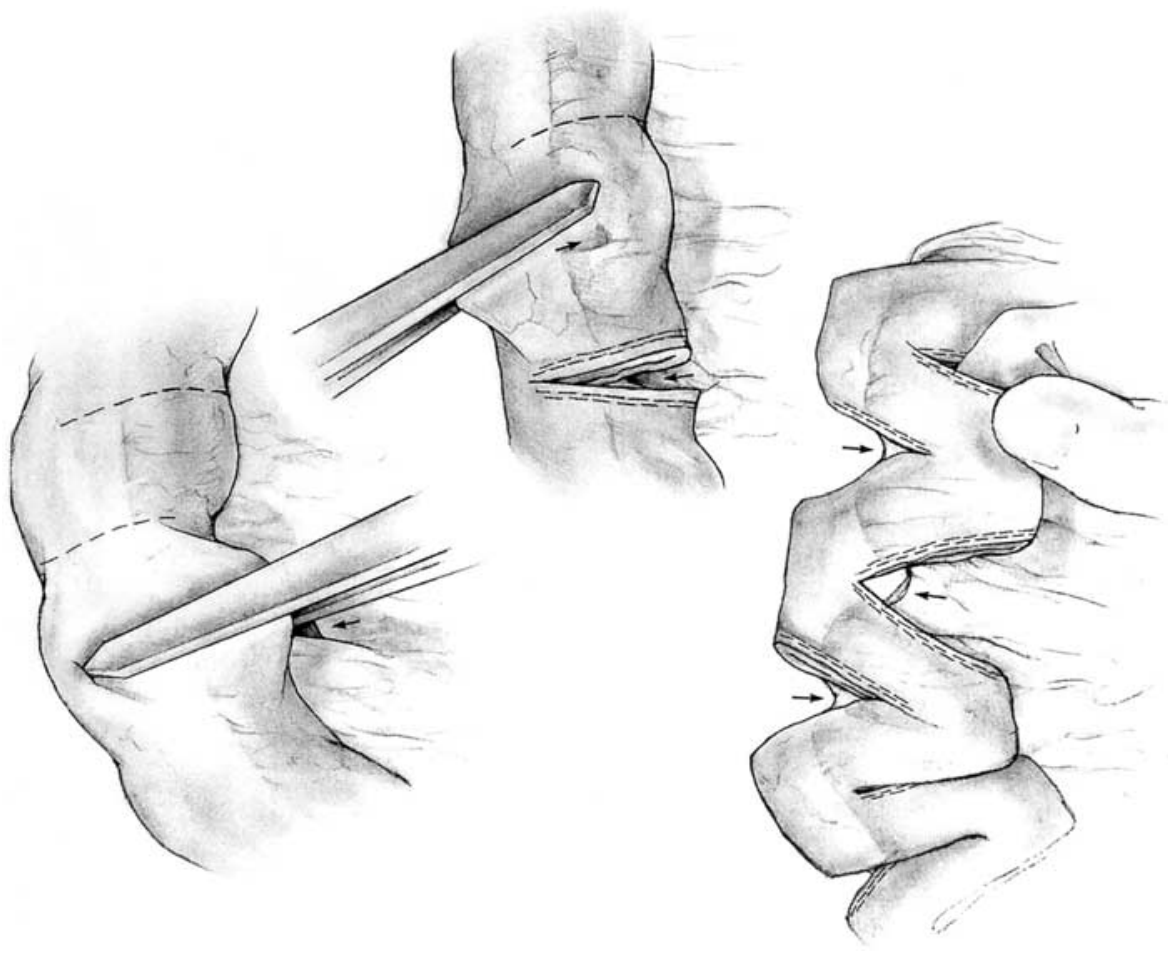

Fig 1. Schematic drawing of the serial transverse enteroplasty procedure. The small arrows show the direction of insertion of the GIA stapler and the sites of the mesenteric defects. The staplers are placed in the $90^{\circ}$ and $270^{\circ}$ orientations using the mesentery as the $0^{\circ}$ reference point. zag-like channel of approximately 2 to $2.5 \mathrm{~cm}$ in diameter (Fig 1 ). This required the creation of a mesenteric defect at each staple line. The staplers were placed from the $90^{\circ}$ and $270^{\circ}$ positions $\left(0^{\circ}\right.$ being the mesenteric border) in all animals except for animal 6 , in which we tried the $0^{\circ}$ and $180^{\circ}$ orientations. To keep the bowel from twisting during placement of the stapler, a sterile marker was used to draw a line exactly along the antimesenteric border of the bowel at the start of the procedure (Fig 2). The bowel segment distal to the anastomosis was used as an in situ self-control for morphometric analysis. All measurements of diameter were taken with the bowel compressed flat and so were actually measurements of half the circumference of the bowel, but for simplicity of discussion, will be referred to as diameter. The laparotomy was closed in layers and animals were fed ad libitum postoperatively.

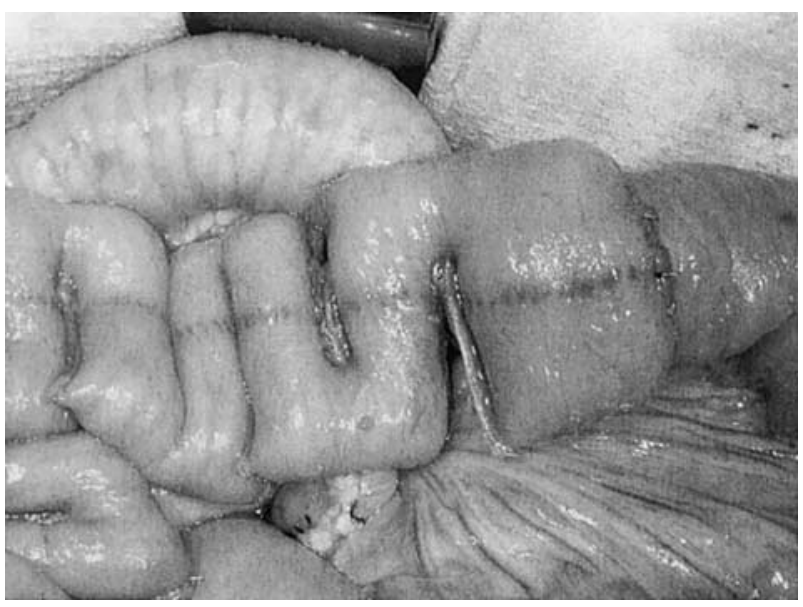

Fig 2. Bowel after the STEP procedure. Note the dotted line marking the antimesenteric border. This keeps the bowel from twisting during placement of the stapler.

\section{Intestinal Motility and Morphometric Analyses}

Six weeks after the STEP procedure, abdominal roentgenograms were performed in all animals 24 hours after oral administration of one gelatin capsule containing 24 radiopaque polyvinyl chloride O-rings of $1 \mathrm{~mm} \times 4.5 \mathrm{~mm}$ (Sitzmarks, Konsyl Pharmaceuticals, Fort Worth, TX). This was followed by oral administration of barium and a contrast abdominal roentgenogram 16 to 24 hours later. Immediately after the last roentgenogram the animals were killed when bowel morphology and dimensions from both the proximal and distal marked segments were examined.

\section{Statistical Analysis}

Statistical analysis was by paired $t$ test. Significance was set at $P$ values of less than .05. Data were expressed as mean \pm SD.

\section{RESULTS}

All 6 animals survived the 2 operative procedures. A summary of the quantitative findings is shown in Table 1. Between the STEP procedure and death, all animals gained weight $(42.5 \pm 3.5 \mathrm{~kg} v 66.7 \pm 3.0 \mathrm{~kg} ; P<$ .0001 ), with no clinical or radiologic evidence of intestinal obstruction. Measurements taken at the second operation showed a good bowel dilatation response to the reversed intestinal segment. The diameter of the proximal dilated bowel increased to an average of $6.2 \pm 0.7$ cm compared with the distal control diameter of $3.6 \pm$ $0.2 \mathrm{~cm}$. The bowel lengthening procedure was performed to achieve a channel size of approximately 2 to $2.5 \mathrm{~cm}$ throughout the length of the intestinal segment. This required approximately 15 to 20 loads of the GIA stapler per animal. The final result is shown in Fig 3. 
Table 1. Summary of Data on Six Pigs That Underwent the STEP Procedure

\begin{tabular}{|c|c|c|c|c|c|c|c|}
\hline \multicolumn{8}{|c|}{ Animal No. } \\
\hline & 1 & 2 & 3 & 4 & 5 & 6 & Mean \pm SD \\
\hline \multicolumn{8}{|l|}{ Weight $(\mathrm{kg})$} \\
\hline Before STEP & 38 & 46 & 43 & 40 & 47 & 41 & $42.5 \pm 3.5^{*}$ \\
\hline Harvest & 70 & 67 & 69 & 63 & 68 & 63 & $66.7 \pm 3.0^{*}$ \\
\hline \multicolumn{8}{|c|}{ Bowel lengths during STEP procedure $(\mathrm{cm})$} \\
\hline Before STEP & 45 & 50 & 50 & 50 & 50 & 50 & $49.2 \pm 2.0$ \\
\hline After STEP & 76 & 78.5 & 77 & 93 & 85 & 87 & $82.8 \pm 6.7$ \\
\hline Immediate increase & 31 & 28.5 & 27 & 43 & 35 & 37 & $33.6 \pm 6.0$ \\
\hline$\%$ Immediate increase & $69 \%$ & $57 \%$ & $54 \%$ & $86 \%$ & $70 \%$ & $74 \%$ & $68 \pm 11 \%$ \\
\hline \multicolumn{8}{|l|}{ Bowel lengths at harvest $(\mathrm{cm})$} \\
\hline Control bowel length & 52 & 45 & 50 & 67 & 57 & 72 & $57.2 \pm 10.4 \dagger$ \\
\hline STEP bowel length & 73 & 69 & 98 & 74 & 73 & 97 & $80.7 \pm 13.1 \dagger$ \\
\hline Final increase & 28 & 19 & 48 & 24 & 23 & 47 & $31.5 \pm 12.7$ \\
\hline$\%$ Final increase & $62 \%$ & $38 \%$ & $96 \%$ & $48 \%$ & $46 \%$ & $94 \%$ & $64 \pm 25 \%$ \\
\hline \multicolumn{8}{|l|}{ Intestinal diameters $(\mathrm{cm})$} \\
\hline Dilated bowel size & 7 & 6 & 6.3 & 7 & 5.5 & 5.5 & $6.2 \pm 0.7$ \\
\hline Initial STEP channel size & 2.5 & 2 & 2 & 2 & 2 & 2 & $2.1 \pm 0.2$ \\
\hline Final STEP channel size & 4.2 & 3.6 & 4.7 & 3.5 & 4.5 & 5.5 & $4.3 \pm 0.7 \ddagger$ \\
\hline Initial control bowel size & 3.5 & 3.6 & 3.9 & 3.5 & 3.4 & 3.5 & $3.6 \pm 0.2$ \\
\hline Final control bowel size & 3.8 & 4.1 & 3.3 & 3.4 & 4.1 & 4 & $3.8 \pm 0.4 \ddagger$ \\
\hline
\end{tabular}

NOTE. All animals gained weight after the STEP, and none showed signs of obstruction. Bowel length was increased immediately and remained significantly longer than control bowel at harvest. Bowel diameter was significantly reduced after the STEP, but at harvest, the STEP channel size and the control bowel channel size were not significantly different.

${ }^{*} P<.0001$

$\dagger P<.01$.

$\ddagger P$, not significant.

The STEP procedure resulted in an immediate increase in length from an average of $49.2 \pm 2.0 \mathrm{~cm}$ to $82.8 \pm 6.7 \mathrm{~cm}$, an increase of $68 \%$. Six weeks after surgery, at death, the lengthened intestinal segment had become practically straight and remained significantly longer than the in situ control $(80.7 \pm 13.1 \mathrm{~cm} v 57.2 \pm$ $10.4 \mathrm{~cm} ; P<.01)$. Interestingly, the STEP channel size increased over the postoperative period from an average of $2.1 \pm 0.2 \mathrm{~cm}$ to $4.3 \pm 0.7 \mathrm{~cm}$, exceeding the radial growth of the distal control bowel $(3.6 \pm 0.2 \mathrm{~cm}$ to $3.8 \pm$ $0.4 \mathrm{~cm}$ ) despite the fact that the reversed intestinal

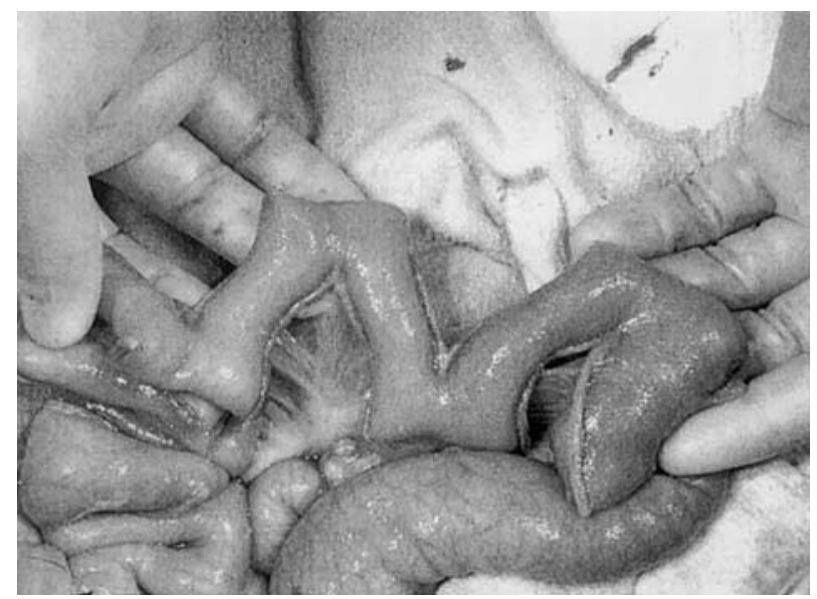

Fig 3. The final result of the STEP is a zig zag-shaped channel of bowel that is longer and narrower than the original dilated loop. segment was removed. Although the bowel lengthening procedure was performed to achieve an intestinal diameter of approximately 2 to $2.5 \mathrm{~cm}$ intraoperatively, there were no significant differences in diameter between the STEP channel and the distal control bowel at death $(4.3 \pm 0.7 \mathrm{~cm} v 3.8 \pm 0.4 \mathrm{~cm} ; P$ value, not significant). We did not notice any significant difference in animal 6 that had the staples applied from the $0^{\circ}$ and $180^{\circ}$ orientations compared with the other 5 animals. Both barium and Sitzmark radiopaque rings were visible by roentgenogram in the rectum of all animals in less than 24 hours.

\section{DISCUSSION}

Short bowel syndrome is a devastating condition that is characterized clinically by an inability to absorb adequate enteral nutrition to sustain normal growth and development. The potential causes of SBS include a variety of congenital and acquired conditions that result in loss of normal bowel mucosal surface area either in utero or postnatally. ${ }^{4}$ The management of SBS was revolutionized with the advent of total parenteral nutrition (TPN). ${ }^{5}$ The use of TPN permits the survival of children who would have otherwise died of the complications of malnutrition. In addition, it gives the remaining bowel time to undergo adaptation. The process of bowel adaptation includes mucosal hyperplasia, which results in increased mucosal surface area. However, this also is accompanied frequently by bowel dilatation that 
results in motility problems as well as bacterial overgrowth in the dilated segments. ${ }^{6}$ Thus, the majority of patients with short bowel syndrome who are TPN dependent suffer from 2 major structural problems, namely, insufficient bowel length and excessive bowel dilatation.

Surgical therapy for SBS is aimed at correcting these 2 anatomic problems. Elegant procedures for increasing bowel length already have been described by Bianchi $^{2}$ and Kimura and Soper ${ }^{3}$. The procedure described by Bianchi $^{2}$ is based on the principle that the mesenteric blood supply to the bowel "splits" as it enters the bowel wall so that the bowel and mesentery can be divided longitudinally while maintaining half of the blood supply to each half of the bowel. The procedure described by Kimura and Soper $^{3}$ is based on allowing time for the antimesenteric border of the bowel to "parasitize" a new blood supply from the liver and abdominal wall musculature before dividing it from the mesenteric half and creating a new tube. Both of these procedures are technically demanding but can result in bowel that is doubled in length with a circumference that is reduced by half. There is some evidence to show that the use of these surgical procedures may allow weaning from TPN. ${ }^{7,8}$

The STEP procedure is based on the anatomic principle that the blood supply to the bowel comes from the mesenteric border of the bowel and traverses the bowel, remaining perpendicular to the long axis of the bowel. Thus, if all staple lines are kept perpendicular to the long axis of the bowel, all segments of bowel should remain well vascularized. Alternating the direction of the stapler from side to side creates a channel of bowel that is both smaller in diameter and longer in length than the original bowel. Thus, this procedure is equivalent in effect to both the Bianchi and the Kimura procedures. However, we feel that the STEP procedure has several theoretic advantages over these other methods. First, the procedure is quite easy to perform. There are no anastomoses, the bowel is never opened, and the mesentery is never jeopardized. Second, the total theoretical increase in length depends on the degree of bowel dilatation and the size of the channel created (Fig 4). With massively dilated segments, one could easily more than double the length of bowel. In our study, we were only able to increase the final length by $64 \%$, but this was with only modestly dilated proximal bowel (less than 2 times the size of the control distal bowel. Third, the degree of tapering is customizable. With a Bianchi procedure, the circumference must be reduced by $50 \%$. With the STEP, the channel size and thus the diameter is determined by the surgeon. We have shown here that the bowel after the STEP procedure tends to grow, so that the channel size can be made smaller than the control distal bowel and thus allow even more lengthening and tapering effect. Lastly, the STEP procedure can be performed in se-

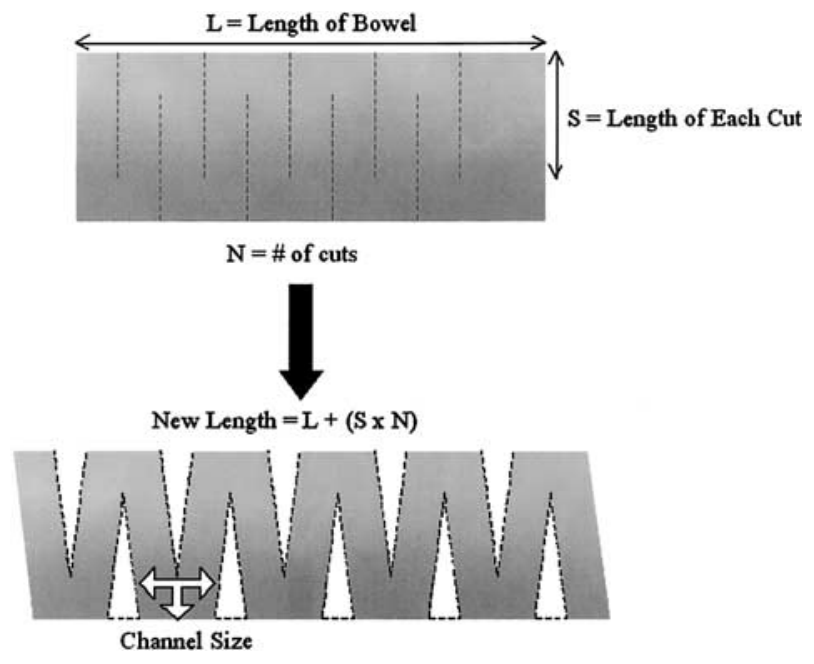

Fig 4. Schematic of the STEP procedure. The formula for the theoretic new length of bowel is calculated based on measurement along one side of the lengthened bowel. The channel size is determined by the length of the staple line and the distance between staple lines. We try to keep the channel size constant along the length of the bowel.

quence after a successful Bianchi procedure, should the Bianchi segments of bowel dilate again. Because the blood supply to the bowel after a Bianchi procedure remains perpendicular to the long axis of the bowel, staple lines that remain perpendicular to the long axis of the bowel should not cause ischemia. This would theoretically allow one to first double the length of the bowel with a Bianchi, followed several months later with a STEP, resulting in an increase in bowel length exceeding 2-fold and possibly reaching 3 to 4 fold or greater, depending on the degree of bowel dilatation.

During our experience in the pig model, we were able to learn several valuable technical points. First, a mesenteric defect should be made at each and every staple site. We initially thought that the mesentery could just be rolled or folded, and we could avoid making multiple mesenteric defects. However, we found that if the defects are not created, the staples tend to be placed at less that $180^{\circ}$ apart, resulting in a series of bowel diverticuli, rather than a smooth zig zag channel. We have not closed any of the small mesenteric defects and have not had any problems with internal hernias in this small series. Second, at autopsy, it appears that the bowel actually straightens out, and, if not for the presence of the staples, it would be difficult to identify the STEP bowel. Third, we have found that keeping the appropriate orientation of the bowel can be challenging because the bowel has a tendency to roll during placement of the stapler. An antimesenteric stripe made with a sterile skin marker at the start of the procedure helps to maintain orientation (Fig 2). The stripe is kept exactly at the midpoint of the 
bowel after it is flattened before application of the stapler. Fourth, we tried the STEP procedure with the $90^{\circ}$ to $270^{\circ}$ orientation as well as the $0^{\circ}$ to $180^{\circ}$ orientation. With the former orientation, a mesenteric defect is required at each staple line, whereas with the latter, a defect is needed only at every other staple line. The bowel appeared to lay better using the $90^{\circ}$ to $270^{\circ}$ orientation in that it seemed less likely to twist or kink, but, overall, there did not seem to be a great difference between the 2 methods. Lastly, at the proximal end of the STEP, the bowel tends to dilate gradually from normal bowel near the duodenum to dilated bowel. To avoid an abrupt change in diameter of the bowel, we start with short staple lines and sequentially increase the length of each staple line keeping the channel at the desired size.
In this report, we have established the technical principles for a novel bowel lengthening procedure that may benefit children with short bowel syndrome and dilated bowel. This method could, however, also be applied to a variety of other select cases in which bowel tapering is desired but in which loss of mucosal surface area might result in malabsorption, such as the case of intestinal atresias with massively dilated proximal bowel. We also have shown that this procedure can be performed safely and easily and that it does not result in intestinal obstruction. However, our porcine model was not a short bowel model; therefore, further studies will be required to assess the clinical efficacy of this procedure and its ultimate role in the management of patients with short bowel syndrome.

\section{REFERENCES}

1. Thompson JS, Langnas AN, Pinch LW, et al: Surgical approach to short-bowel syndrome. Ann Surg 222:600-607, 1995

2. Bianchi A: Intestinal loop lengthening-A technique for increasing small intestinal length. J Pediatr Surg 15:145-151, 1980

3. Kimura K, Soper RT: A new bowel elongation technique for the short-bowel syndrome using the isolated bowel segment Iowa models. J Pediatr Surg 28:792-294, 1993

4. Andorsky DJ, Lund DP, Lillehei CW, et al: Nutritional and other postoperative management of neonates with short bowel syndrome correlates with clinical outcomes. J Pediatr 139:27-33, 2001

5. Dudrick SJ, Wilmore DW, Vars HM, Rhoads JE: Long-term total parenteral nutrition with growth, development, and positive nitrogen balance. Surgery 64:134-142, 1968

6. Gambarara M, Ferretti F, Papadatou B, et al: Intestinal adaptation in short bowel syndrome. Transplant Proc 29:1862-1863, 1997

7. Georgeson K, Halpin D, Figueroa R, et al: Sequential intestinal lengthening procedures for refractory short bowel syndrome. J Pediatr Surg 29:316-321, 1994

8. Figueroa-Colon R, Harris PR, Birdsong E, et al: Impact of intestinal lengthening on the nutritional outcome for children with short bowel syndrome. J Pediatr Surg 31:912-916, 1996 